\title{
Designing Solutions for Electrospinning of Poly(ionic liquid)s
}

\author{
Elinor Josef* ${ }^{*}$ and Ryan Guterman ${ }^{(0)}$ \\ Department of Colloids Chemistry, Max Planck Institute of Colloids and Interfaces, Research Campus Golm, Am Mühlenberg 1, \\ 14476 Potsdam, Germany
}

Supporting Information

ABSTRACT: Production of polyelectrolyte fibers via electrospinning is more challenging compared to neutral polymers because of their ionic nature. In this paper, a comprehensive approach to achieve poly(ionic liquid) fibers is presented. We show that small-angle X-ray scattering is a valuable tool to provide a deeper understanding of the structure of the polymer in solution and its relation to spinnability. We found that in dimethylformamide, the poly(ionic liquid) acted like a conventional polyelectrolyte with disassociated ions, and attempts to electrospin it resulted in bead formation. When

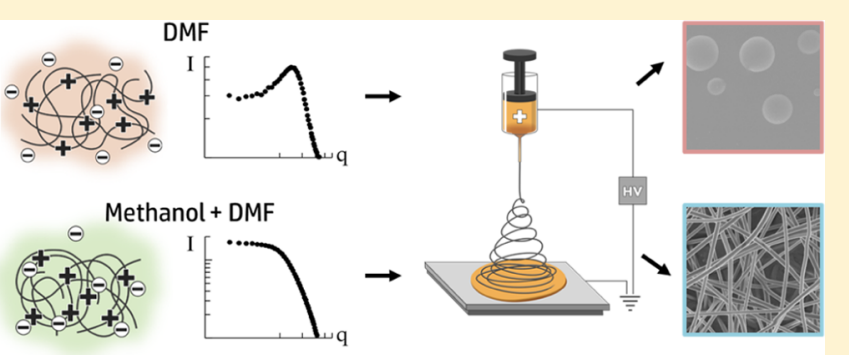
methanol was added, the polymer structure was more neutral-like and fibers were successfully formed. Thus, tuning the solvent of the electrospinning solution could induce a change in the structure of the polyelectrolytes in the solution to a more neutral structure that favors fiber production. This approach could facilitate the development of future electrospun polyelectrolyte fibers.

\section{INTRODUCTION}

One-dimensional nanofibers have a high surface-to-volume ratio and controllable porosity, making them promising candidates in tissue engineering, catalysis, energy storage, water treatment, fiber reinforcement in composites, and more. ${ }^{1-5}$ Nanofibers can be produced using different methods, including self-assembly, drawing, solution blow spinning, template-based synthesis, and electrospinning. ${ }^{6}$ From these methods, electrospinning is the most established and widely used because of its simplicity, scalability, and top-down approach. $^{6,7}$ This technique allows for the production of continuous fibers with interconnected porosity to form a freestanding mat. The first large-scale commercial application of electrospun fibers dates back to the early 1980s when they were used in air filters. ${ }^{8,9}$ Starting in the mid-1990s, the potential of electrospinning for the production of nanofibers was noticed by the academic community, which spawned new research directions to take advantage of this material architecture. To date, more than 100 polymers have been processed into fibers by means of electrospinning. ${ }^{10}$ The polymers could be the final fiber material or a carrier for other materials such as metals and ceramics. ${ }^{11}$ Of these polymers, polyelectrolytes are among the most difficult to electrospin because of their different properties in solution compared to neutral polymers. Yet, they have promising applications as scaffolds for tissue engineering and drug carriers, where many of the biocompatible polymers used are polyelectrolytes (e.g., alginate, polylactic acid, collagen, chitosan, and hyaluronic acid), ${ }^{2,12}$ making their electrospinning to a scaffold that mimics the natural extracellular matrix a vibrant area of research. Polyelectrolytes are also favorable in applications that require ion conductivity, for example, in solid-state electrochromic devices as the electrolytes ${ }^{13}$ and as proton conductors in membranes. ${ }^{14}$ Finding an approach to shorten the development process for material fabrication would accelerate their implementation in these fields.

In an electrospinning process, a polymer solution is pumped through a spinneret, which is subjected to high voltage. If the electrical forces formed by the high voltage are high enough to overcome the surface tension of the polymer solution, a jet erupts from the spinneret. As the jet stretches and undergoes a whipping motion, the solvent evaporates, the jet solidifies, and fibers are deposited on the collector. Many parameters influence the electrospinning process, including ambient parameters (temperature and humidity), process parameters (e.g., flow rate, voltage, distance from tip to collector, and collector type), and solution parameters (e.g., polymer concentration, solvent or solvents, viscosity, conductivity, and surface tension). ${ }^{15,16}$ The extent to which each parameter determines the spinning ability is still debated, as studies sometimes show contradicting results. ${ }^{17,18}$ Although there is much research effort focused on understanding the mechanism of electrospinning and the influence of the different parameters, ${ }^{19-22}$ theoretical models that encompass all variables are still not developed. ${ }^{2,4}$ There are general guidelines pertaining the solution properties to its spinnability; ${ }^{23}$ but there is still a need to expand existing principles to better predict spinning conditions for new polymers.

Polyelectrolytes are often more challenging to spin in comparison to neutral polymers for a number of reasons. First,

Received: April 5, 2019

Revised: June 9, 2019

Published: July 8, 2019 


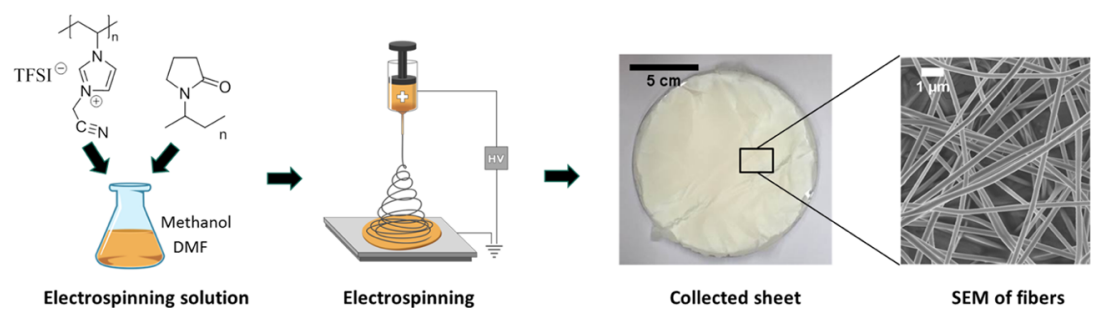

Figure 1. Scheme of the electrospinning process. A solution of PIL and PVP was prepared in DMF and methanol and electrospun to form fibers.

the relatively low mobility of the polyelectrolyte may cause instabilities in the electrospinning jet. $^{24}$ Second, many polyelectrolytes are only soluble in water, which is not ideal because of the high surface tension of water. Third, ion disassociation in some solvents leads to high electrical conductivity of the spinning solution, ${ }^{25}$ which beyond a critical value (depending on the system used) hinders electrospinning. ${ }^{4,26,27}$ Furthermore, the repulsion between the charged groups leads to different scaling and rheological behaviors. For example, a prerequisite to achieve bead-free fibers is that the polymer concentration in the spinning solution be above the entanglement concentration $\left(C_{\mathrm{e}}\right)$, more than 2.5 entanglements per chain in a good solvent. ${ }^{28}$ For polyelectrolytes, the required concentration is estimated to be more than $8 C_{\mathrm{e}}{ }^{24}$ at which point the viscosity could be too high to flow through the spinneret. A possible way to overcome the obstacle of electrospinning polyelectrolytes is to make them more neutral de-facto by screening some of the charges; a few studies have investigated this route. ${ }^{27,29}$ Long et al. ${ }^{24}$ showed that by adding salt to the spinning solution, both the viscosity of the solution and the concentration required to achieve fibers decreased. Similarly, Green and co-workers studied the electrospinning of segmented imidazolium ionenes in acetonitrile $(\mathrm{ACN})$ and observed that when the charge density is low, a lower value of $C / C_{e}$ is required for the onset of fiber formation. $^{30}$

Substantial research is dedicated to the structure of polyelectrolytes in solution; however, our understanding of their behavior in solution is not as developed as for neutral polymers. Historically, considerable attention was given to characterize polyelectrolytes in aqueous solutions compared to nonaqueous solutions because of their relevance in biology (proteins, DNA, and polysaccharides) and functions in polymer chemistry. ${ }^{31-33}$ Not all charged polymers exhibit polyelectrolyte behavior in organic solvents; ${ }^{34}$ however, the polyelectrolyte behavior in nonaqueous media has been observed in both polar ${ }^{35-39}$ and nonpolar ${ }^{32}$ solvents. Understanding the behavior of polymers in the spinning solution could lead to a more targeted design of materials for spinning. However, most research for developing electrospinning solutions focuses on measuring the viscosity, conductivity, and surface tension of the polymer solution, although they are not enough to give a complete picture. ${ }^{40}$ Small-angle scattering of neutrons or X-rays is used to investigate the conformation of neutral and charged polymers in solution ${ }^{41-43}$ but is infrequently applied to study the relationship between electrospinnability of solutions and the local and global conformation of the polymer. ${ }^{40,44,45}$

Poly(ionic liquid)s (PILs) are a class of polyelectrolytes that feature an ionic liquid (IL) species in each repeating unit, which may or may not be synthesized directly from IL monomers. ${ }^{46}$ PILs extend the useful properties of ILs, such as chemical versatility, ion conductivity, high thermal stability, and high carbonization yields, to a polymer form, which expands the utility of ILs beyond their conventional applications. ${ }^{47-52}$ Knowing how to process PILs to create new materials is essential to their broader application; electrospinning provides a unique architecture that is not possible with other processing methods. The one-dimensional fibers together with the flexible chemistry of PILs could enable production of ion conductive membranes, functionalization of membranes, modification of surface wetting properties for oil/ water separations, and heteroatom doping for future carbon fibers. However, there are only a few examples of PIL fibers produced using electrospinning, ${ }^{48,53-56}$ possibly because of their typical low molecular weight and highly charged nature. Unlike many polyelectrolytes, PILs are soluble in many conventional organic solvents and are thus good candidates to obtain electrospun polyelectrolyte fibers.

In this context, we developed an approach for electrospinning polyelectrolytes using the solvent composition as a way to affect the polyelectrolyte behavior in the solution. A scheme of the electrospinning process is shown in Figure 1. The polyelectrolyte used was poly(3-cyanomethyl-1-vinylimidazolium bis(trifluoro methanesulfonyl)imide) (PIL) (Figure S1), a lipophilic PIL which allows electrospinning using organic solvents rather than water. Neutral polymers such as polyvinylpyrrolidone (PVP), poly(ethylene oxide), or poly(vinyl alcohol) (PVA) $)^{48,57}$ are often added to polyelectrolyte solutions in order to increase chain entanglements without substantially increasing the viscosity; ${ }^{58}$ we used PVP as the supporting polymer. We studied the effect of using a mixture of solvents, dimethylformamide (DMF) and methanol, on the electrospinning performance and recognized that this mixture was key to achieve fibers. Using small-angle X-ray scattering (SAXS), we observed that the structure of PIL was different in each solvent. In DMF, the PIL showed the characteristic polyelectrolyte scattering peak, whereas in methanol, the peak disappeared, indicating a more neutral behavior. This more neutral structure was met with improved spinnability. Thus, we show for the first time that altering the solvent composition could be an approach to achieve better spinnability, by driving the behavior of the polyelectrolyte to a more neutral-like polymer. This observation could allow for smarter design of electrospinning solutions and be implemented in future development of polyelectrolyte fibers.

\section{RESULTS AND DISCUSSION}

The molecular weight of a polymer is an important parameter for electrospinning. Sufficiently high molecular weight is required to allow for the entanglements of the polymer chains; however, a molecular weight that is too high could inhibit fiber formation. ${ }^{59}$ Poly(3-cyanomethyl-1-vinylimidazolium bis(trifluoro methanesulfonyl)imide) was synthesized according 

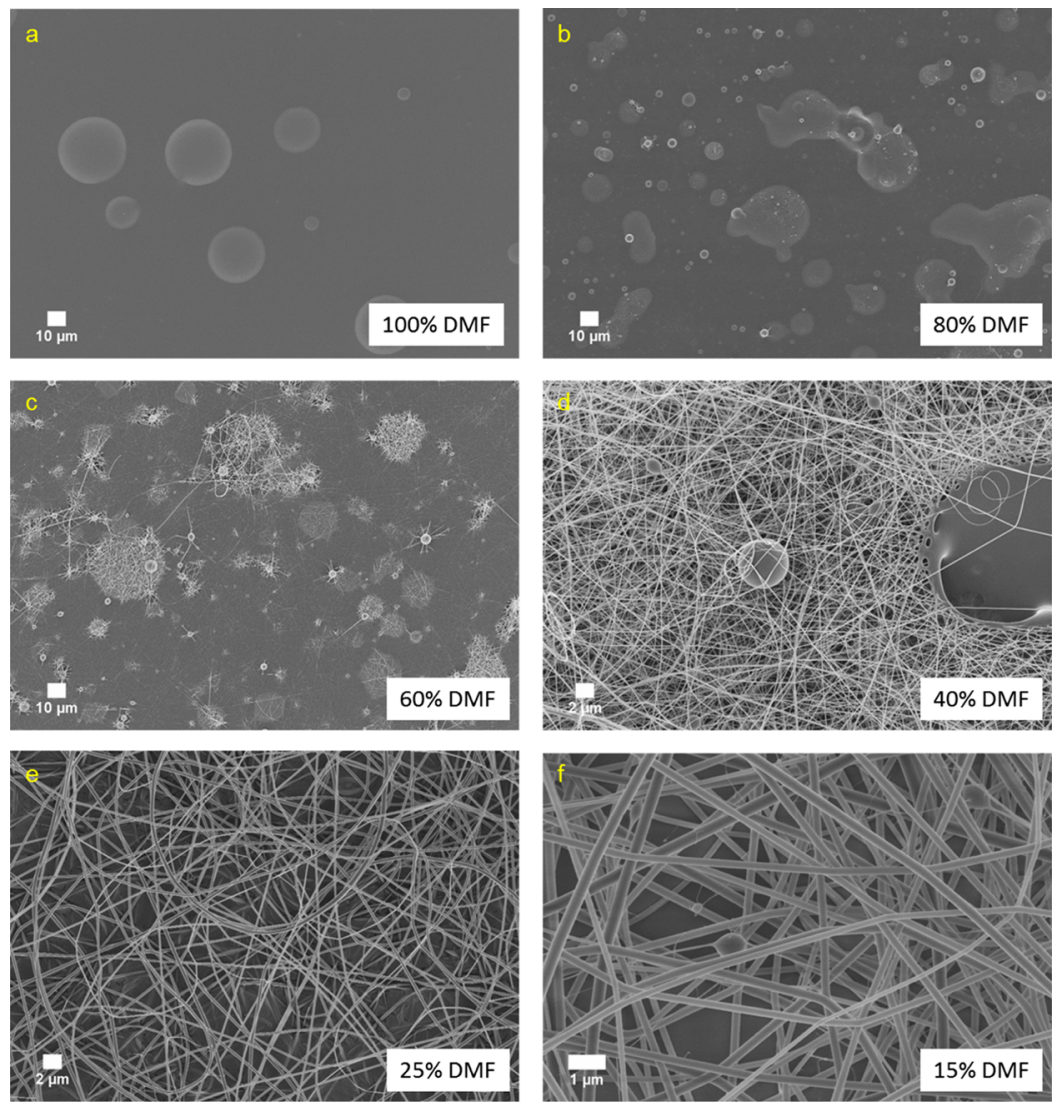

Figure 2. Scanning electron microscopy (SEM) micrographs of electrospinning MIX-X solutions. (a) MIX-100, (b) MIX-80, (c) MIX-60, (d) MIX-40, (e) MIX-25, and (f) MIX-15.
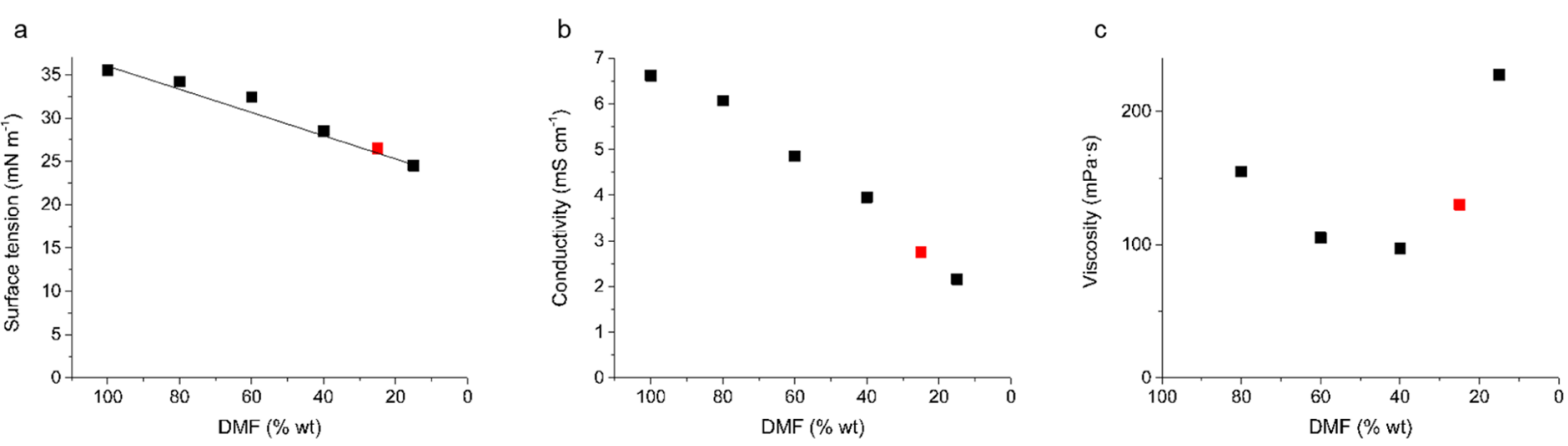

Figure 3. Surface tension (a), conductivity (b), and viscosity (c) of MIX-X. X-axis is the weight fraction of DMF in the DMF and methanol mixture. The red points are MIX-25, the best solution for electrospinning. The line in (a) represents the calculated surface tension of the mixture of solvents without any solute. In (c), the viscosity of MIX-100 was visibly higher than MIX-15, and it was not possible to measure it in the same instrumental setup.

to a previous procedure ${ }^{60}$ which resulted in highly viscous solutions that were not suitable for electrospinning. Performing the polymerization in more dilute solutions lowered the molecular weight to $110 \mathrm{~kg} \mathrm{~mol}^{-1}$ from the original $315 \mathrm{~kg}$ $\mathrm{mol}^{-1}$ and resulted in solutions with lower viscosities. The reaction conditions are detailed in the Materials and Methods. The lower molecular weight poly(3-cyanomethyl-1-vinylimidazolium bis(trifluoro methanesulfonyl)imide) (PIL) was then electrospun from a solution containing $20 \mathrm{wt} \% \mathrm{PIL}$ and 4 wt \% PVP, with DMF or a mixture of DMF and methanol as solvents (Figure 1). A minimal amount of PVP was used to achieve spinnable solutions. The effect of the solvent ratio on the spinning outcome was investigated, while keeping other process parameters constant (high voltage $20 \mathrm{kV}$, flow rate 1.2
$\mathrm{mL} \mathrm{h}^{-1}$, and distance from tip to collector of $\left.10 \mathrm{~cm}\right)$. Spinning solutions are labeled MIX-X, where $X$ represents the weight percentage of DMF with respect to the weight of the mixture of solvents. In a similar manner, solutions with only $20 \mathrm{wt} \%$ PIL or 4 wt \% PVP are written as PIL- $X$ and PVP-X, respectively. Electrospinning MIX-100 resulted in the formation of only particles (Figure 2a). Upon addition of methanol, a mixture of fibers and particles was observed (MIX80 and MIX-60, Figure 2b,c). For MIX-40, mostly fibers were produced; however, small beads and large droplets containing trapped solvent destroyed the fibers instantly, prohibiting the collection of a robust material (Figure $2 \mathrm{~d}$ ). The electrospinning of MIX-25 was stable, and only fibers were formed (Figure 2e). Increasing the methanol content further (MIX- 
15) produced spinning instabilities and some beaded fibers because of rapid solidification at the tip of the cone (Figure $2 \mathrm{f})$. For this reason, 25 wt \% DMF was found to be the appropriate concentration for producing PIL fibers. Fibers from MIX-25 were collected for $1 \mathrm{~h}$, during which the process was stable, without any dripping or build-up of fibers toward the spinneret as is sometimes observed for PILs, ${ }^{53}$ and a $14 \mathrm{~cm}$ diameter mat was obtained (Figure S2).

The ideal polymer solution for electrospinning is dependent, among other things, on the combined contributions of viscosity, conductivity, and surface tension. ${ }^{61-63}$ We first examined the surface tension of solutions MIX-100 to MIX-15 and found that the surface tension of the different spinning solutions is governed by the surface tension of the mixture of solvents only and did not change significantly when PIL and PVP were added (Figure 3a). A previous study on PILs and PVP in either DMF or methanol showed that some PILs change the surface tension of solution, whereas other types of PILs do not. ${ }^{48}$ In addition, the value of surface tension, between that of DMF and that of methanol, did not seem to have an effect on the spinning outcome.

Although the PIL did not affect the surface tension, it increased the electrical conductivity of the solution significantly. MIX-100 to MIX-15 and PIL-100 to PIL-15 solutions had an electrical conductivity in the range of $2-7 \mathrm{mS} \mathrm{cm} \mathrm{cm}^{-1}$, 100 times higher than PVP-100 to PVP-15, whose conductivities were $10-13 \mu \mathrm{S} \mathrm{cm} \mathrm{cm}^{-1}$ (Table 1). The

Table 1. Viscosity and Conductivity of Solutions MIX-X, PIL- $X$, and PVP- $X$ with Different Amounts of DMF

\begin{tabular}{cccccccc} 
& \multicolumn{3}{c}{ viscosity $(\mathrm{mPa} \cdot \mathrm{s})$} & & \multicolumn{3}{c}{ conductivity $\left(\mathrm{mS} \mathrm{cm}^{-1}\right)$} \\
\cline { 2 - 4 } \cline { 6 - 8 } \% DMF & MIX- $X$ & PIL- $X$ & PVP- $X$ & & MIX- $X$ & PIL- $X$ & PVP- \\
100 & $>227$ & 15.6 & 10.9 & & 6.6 & 7.4 & $<0.01$ \\
25 & 130 & 4.7 & 8.3 & & 2.7 & 3.4 & 0.013 \\
15 & 227 & 4.4 & 8.7 & & 2.2 & 2.8 & 0.013
\end{tabular}

${ }^{a}$ The weight percent of DMF from the mixture DMF + methanol.

conductivity of the pure solvents was below the sensitivity of the probe $\left(<10 \mu \mathrm{S} \mathrm{cm}^{-1}\right)$. Addition of PVP to PIL solutions decreased the electrical conductivity by $10-25 \%$ (Table 1 ), which we attribute to the significant increase in viscosity, leading to reduced charge mobility. Increasing the conductivity of neutral polymer solutions generally leads to the formation of fibers over beads because sufficient free charges can move to the surface of the jet, thus increasing the net charge density and initiating the Taylor cone. ${ }^{64}$ For polyelectrolytes such as PILs, the solution already possesses a high conductivity, ${ }^{65}$ which may hinder Taylor cone formation. ${ }^{66}$ Although it is simple to increase the conductivity of neutral polymer solutions by adding an electrolyte, decreasing the conductivity is more challenging. We found that altering the solvent composition decreases the conductivity of the solutions with PIL by up to three times. PIL solutions possessing lower conductivity favored spinning; MIX-solutions with decreasing amounts of DMF were less conductive (Figure $3 \mathrm{~b}$ ) and produced more fibers.

Next, we examined the viscosity of MIX-100 to MIX-15 and whether it is a defining parameter for electrospinning of PIL. Solutions MIX-80 and MIX-60 possessed a viscosity similar to MIX-25 (Figure 3c), yet fibers were only achieved for the latter case. This could suggest that the high conductivity of the solutions dominates the spinnability rather than the viscosity. The viscosities of MIX-100 to MIX-15 solutions are 2 orders of magnitude higher than the viscosities of the individual components solutions, PIL-100 to PIL-15 and PVP-100 to PVP-15, which could indicate a transition to the semidilute entangled regime. The viscosity of PIL-X decreased as methanol content increased, whereas for PVP solutions, the viscosity of PVP-100 and PVP-15 is higher than that of PVP25 (the viscosity of PVP-0 was $9.3 \mathrm{mPa} \cdot \mathrm{s}$, higher than that of PVP-15). The phenomenon of minimum viscosity at MIX-40 could thus be related to the PVP itself; however, the effect is more pronounced when PIL is present. One possible explanation for the higher viscosity of PIL in DMF compared to methanol is the presence of more electrostatic charges. It is commonly observed for polyelectrolytes that upon salt addition, the charges are screened and the viscosity decreases. ${ }^{67}$ Consistent with this observation, the viscosity of MIX-100 was higher than that in MIX-15, which is less charged.

The local and global conformation of the polymer in different solvents may affect the spinning behavior. For example, electrospinning of bovine serum albumin (BSA) in water resulted in spray, but when a denaturing agent was added, the conformation of BSA changed and fibers were formed. ${ }^{44}$ This conformational change was detected using SAXS, a direct method to study the structure of polymer in the solution. Upon addition of the denaturing agent, the electrostatic peak disappeared, suggesting that the conformation of BSA changed to a random coil. These results show that by forcing different structures of the macromolecule, it is
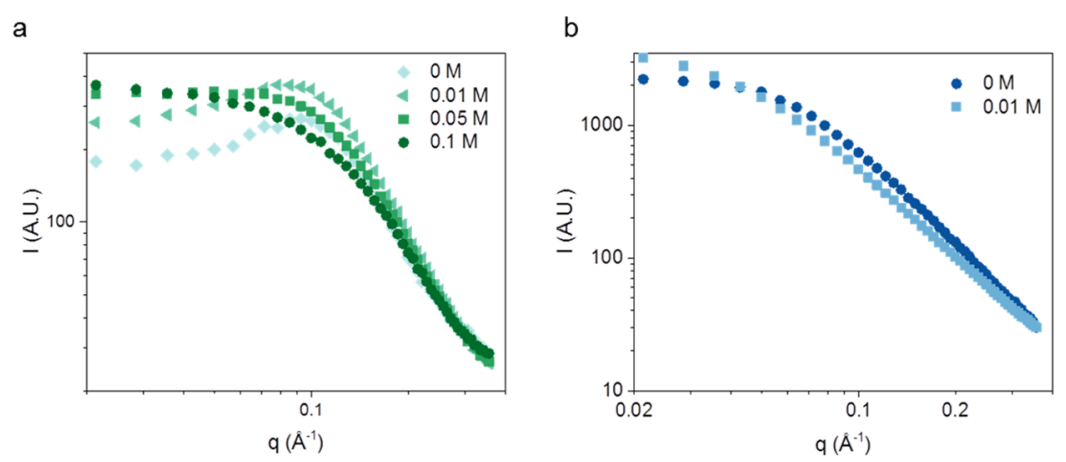

Figure 4. SAXS plots of $105 \mathrm{mg} \mathrm{mL}^{-1}$ PIL solutions with varying concentrations of salt, intensity (arbitrary units) vs scattering vector $q$. (a) PIL in DMF, with LiTFSI $0 \mathrm{M}$ (blue diamond), $0.01 \mathrm{M}$ (left-pointing green triangle), $0.05 \mathrm{M}$ (green square), and $0.1 \mathrm{M}$ (green circle). (b) PIL in methanol, with LiTFSI $0 \mathrm{M}$ (blue circle) and $0.01 \mathrm{M}$ (blue square). 


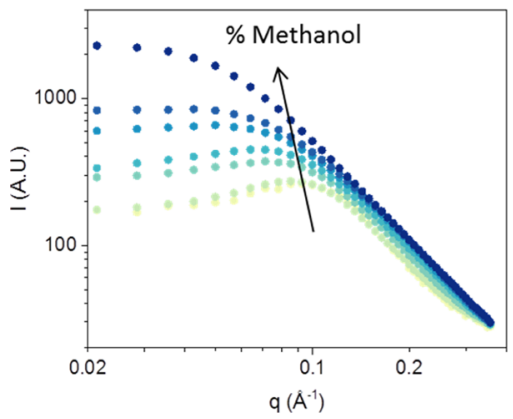

b

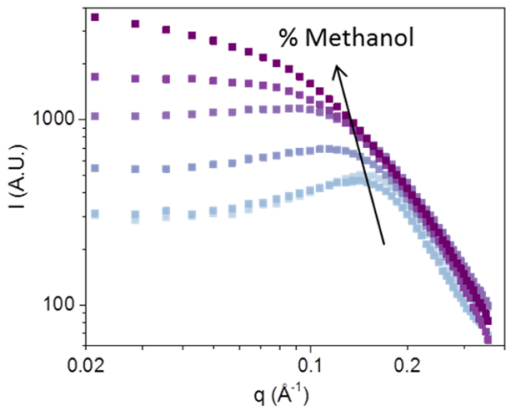

Figure 5. SAXS patterns of (a) $105 \mathrm{mg} \mathrm{mL}^{-1} \mathrm{PIL}$ in mixtures of DMF and methanol, with weight ratios of DMF/methanol from bottom to top 100:0, 80:20, 60:40, 40:60, 25:75, and 15:85 (b) from bottom to top MIX-100, MIX-80, MIX-60, MIX-40, MIX-25, and MIX-15.

possible to obtain spinnable solutions. To determine if this is the case for PIL, we used SAXS to probe the nanostructure of the different solutions. First, we looked into the pristine system, where PIL is dissolved at a fixed molarity and only the composition of the solvent changes. Next, we measured the more complex spinning solutions, containing both PIL and PVP, where both the molarity of the polymers and the solvent composition changed. The conventional nomenclature for polymer concentration is different in electrospinning research and in fundamental polymer structure research, which also demonstrates a basic obstacle to the comparison of studies of the two fields. In electrospinning, the concentration of polymers and other components in the spinning solutions is routinely expressed as weight percent. In studies looking into the structure of polymers in solutions on the other hand, the typical units used are mass or molar concentration (e.g., milligram per millilitre). Therefore, in the following section, we will describe the polymer solutions in terms of mass concentration. A solution of $105 \mathrm{mg} \mathrm{mL}^{-1} \mathrm{PIL}$ (which corresponds to $10 \mathrm{wt} \%$ in DMF) was examined first because this concentration is attainable in both DMF and methanol, allowing the structure in each solvent to be examined. The scattering of $105 \mathrm{mg} \mathrm{mL}^{-1}$ PIL in DMF revealed a structure peak, likely originating from electrostatic interactions between polymer chains (Figure 4a). When the structure peak is a result of electrostatic interactions, screening these interactions causes the peak to decrease in intensity and with enough screening to disappear. A simple way to screen the electrostatic interactions is by the addition of salt. The PIL itself was found to contain very low amounts of salt impurities, measured using inductively coupled plasma optical emission spectrometry (ICP-OES) (details in the Materials and Methods). Following this, we added different concentrations of the salt bis(trifluoromethane)sulfonimide lithium salt (LiTFSI) to 105 $\mathrm{mg} \mathrm{mL}{ }^{-1}$ PIL in DMF and observed the scattering patterns (Figure 4a). Indeed, with greater amounts of added salt, the peak diminished in intensity and disappeared, indicating screening of electrostatic interactions. Thus, the interaction peak is the characteristic polyelectrolyte peak, representing disassociation of the counterions in DMF. An electrostatic peak was observed before for polystyrene sulfonic acid in dimethyl sulfoxide (DMSO) and ethanol. ${ }^{35}$ At the same PIL concentration in methanol, a peak was not observed (Figure 4b), suggesting that in methanol, PIL is more neutral-like. However, the existence of electrostatic interactions between PIL chains in methanol cannot be ruled out, even without the appearance of a peak. LiTFSI $(0.01 \mathrm{M})$ caused a change in the scattering pattern (Figure $4 \mathrm{~b}$ ), illustrating that the salt does induce a change in the conformation of the polymer. At $0.05 \mathrm{M}$ LiTFSI, the PIL precipitated, demonstrating the importance of the charge on the solubility of PIL in methanol. Furthermore, this suggests that methanol is a poor solvent for the PIL backbone because electrostatic interactions are important for the solubility of the PIL.

To investigate whether the behavior observed in PIL solutions is similar to the behavior in solutions containing both PVP and PIL, we measured the scattering of MIX-25. We found that the scattering of the polymer blend is controlled by the scattering of PIL itself because the scattering from PVP itself is negligible in comparison (Figure S3). It is well understood that when two non-interacting components are mixed, the scattering from the mixture equals the sum of scattering of each component. ${ }^{68}$ This sum for PVP and PIL (black line in Figure S3) is similar to the scattering of MIX-25, indicating that PVP does not have a significant effect on the structure of the PIL at length scales of the chain radius, persistence length, or correlation length. We note that thermal gravimetric analysis (TGA) of the spun fibers indicates that there is an interaction between PVP and PIL in the fiber form (Figure S4).

Next, different ratios of DMF and methanol were mixed with $105 \mathrm{mg} \mathrm{mL}^{-1}$ PIL. As the ratio of methanol to DMF increased, the scattering peak shifted to lower $\mathrm{q}$ values and diminished (Figure 5a). Scattering patterns of solutions MIX-100 to MIX15 (Figure $5 b$ ) exhibited a similar trend of diminished peak as methanol content increased. Hence, the PIL undergoes a change in the different solvent ratios, switching from a polyelectrolyte behavior in DMF to a more neutral polymer behavior in methanol. This is in line with the observed decrease in electrical conductivity as methanol content increases, which indicates a smaller disassociation degree of the counterion. More neutral behavior could be favorable to produce fibers by electrospinning because the electrical conductivity is lower, as well as the concentration of polymer required for onset of fiber formation. Long et al. found that spinning was facilitated by adding salt to polyelectrolyte solutions, thereby screening the charges and shifting the viscoelastic behavior from polyelectrolyte toward a neutral polymer. ${ }^{24}$ However, as much as $50 \mathrm{wt} \% \mathrm{NaCl}$ was required to achieve neutral polymer behavior, which also increased the conductivity of the solution by an order of magnitude. In our case, we found that by changing the solvent composition, it was possible to access a more neutral behavior of the polymer, where there was improved spinnability of this PIL. Future work should be conducted to investigate whether this approach can be applied to other PILs and polyelectrolytes. Nonetheless, 
small-angle scattering can be a tool to investigate the structure of polymers in different spinning solutions. We suggest that SAXS could serve as a guide for solvent choice and be the first step before screening different polymer concentrations, thus aiding in eliminating some of the many parameters investigated during the development process of fibers from new materials.

\section{CONCLUSIONS}

A comprehensive approach to achieve spinnable PIL solutions was demonstrated. It was found that low-molecular-weight PIL supported by a long neutral polymer circumvent the viscosity problems associated with the high-molecular-weight PIL. In addition, a solvent mixture of DMF and methanol was crucial to obtain a stable process and beaded-free fibers. Starting from DMF and increasing methanol content, the ratio of fibers to beads grew, until only fibers were produced at 25:75 DMF/ methanol $(w / w)$. Higher methanol fractions resulted in exceedingly high evaporation rates which resulted in solidification of the solution at the tip of the spinneret. Although there are many parameters that affect the spinnability, we showed that conductivity is an important parameter for PIL spinnability and that solvent composition is an approach to achieve appropriate conductivity. Increasing concentration of methanol in the mixture decreased the counterion disassociation degree, which in turn affected the structure of the chains in solution. SAXS was used to detect the changes in the structure, showing that PIL in DMF behaves like a polyelectrolyte, whereas in methanol, charges are screened and the polymer assumes a more neutral structure. Understanding the behavior of PIL in mixed solvents is not trivial, and further experiments should be carried out to investigate the extent of contribution of other parameters, such as the quality and volatility of the solvent, to the spinnability of these solutions. However, changing the solvent composition could be an approach to access a more neutral behavior of the polymer chains, achieve better spinnability, and contribute to the development of future polyelectrolyte fibers.

\section{MATERIALS AND METHODS}

PVP (1.3 MDa), 1-vinylimidazole, and bromoacetonitrile were supplied from Alfa Aesar. All solvents were of analytical grade and supplied from Merck: DMF, methanol, DMSO, acetone, ACN, and diethyl ether. LiTFSI was from IoLiTech. All materials were used as received except azobisisobutyronitrile (AIBN), which was recrystallized in methanol.

Synthesis. IL-Monomer Synthesis. Synthesis of PIL followed a previously described procedure. ${ }^{60}$ 1-Vinylimidazole $(20.0 \mathrm{~g}, 212$ $\mathrm{mmol})$ and butylated hydroxytoluene $(0.1 \mathrm{~g}, 0.45 \mathrm{mmol})$ were dissolved in ACN $(200 \mathrm{~mL})$ in a round bottom flask. Bromoacetonitrile $(16.2 \mathrm{~mL}, 27.9 \mathrm{gr}, 232 \mathrm{mmol})$ was slowly added to the solution and stirred for further $2 \mathrm{~h}$ and then heated to $45^{\circ} \mathrm{C}$ for $16 \mathrm{~h}$. The solution was then cooled to room temperature, the ACN discarded, and the white precipitate washed with diethyl ether $(3 \times 100 \mathrm{~mL})$. After drying in a vacuum oven, a white powder was isolated and identified as the IL monomer, 3-cyanomethyl-1-vinylimidazolium bromide (CMVImBr) (33.5 g, 70\%).

Polymer Synthesis. IL monomer $(6.0 \mathrm{~g}, 28 \mathrm{mmol})$ was dissolved in DMSO $(240 \mathrm{~mL})$ in a $500 \mathrm{~mL}$ round bottom flask. After the monomer was dissolved, the AIBN initiator $(120 \mathrm{mg}, 0.731 \mathrm{mmol})$ was added. The mixture was purged with nitrogen for $30 \mathrm{~min}$ and then heated to $70{ }^{\circ} \mathrm{C}$ and stirred for $16 \mathrm{~h}$. After cooling to room temperature, the solution was slowly added to stirring acetone (400 $\mathrm{mL})$, resulting in the formation of a white oil. The solvent was then decanted and the oil dissolved in DMSO $(60 \mathrm{~mL})$ and precipitated in acetone $(200 \mathrm{~mL})$, followed by rinsing of the oil with acetone $(4 \times$
$100 \mathrm{~mL}$ ) to remove residual DMSO. With each consecutive wash, the oil began to form a beige precipitate. After drying in a vacuum oven $\left(75^{\circ} \mathrm{C}, 16 \mathrm{~h}\right)$, a beige powder was isolated and identified as a CMVImBr polymer $(4.7 \mathrm{~g}, 78 \%)$. The number averaged molecular weight $\left(M_{\mathrm{n}}\right)$ was measured by gel permeation chromatography and found to be $70 \mathrm{kDa}$ with a polydispersity index of 2.4 .

lonic Exchange. The CMVImBr polymer $(4.0 \mathrm{~g}, 19 \mathrm{mmol})$ was solubilized in deionized water $(400 \mathrm{~mL})$. LiTFSI $(5.63 \mathrm{~g}, 20 \mathrm{mmol})$ was solubilized in deionized water $(40 \mathrm{~mL})$. The polymer solution was added slowly to the salt solution while mixing. The resultant mixture was stirred for $1 \mathrm{~h}$ before filtering the solution using Por. 4 Buchner filter. The polymer was washed five times with water and dried in a vacuum oven overnight at $70{ }^{\circ} \mathrm{C}(5.6 \mathrm{~g}, 72 \%)$. A ${ }^{1} \mathrm{H}$ NMR spectrum of the polymer can be found in the Supporting Information (Figure S5). The concentration of residual lithium in the polymer was measured using ICP-OES (Optima 8000, PerkinElmer with multi elemental standard). It was found to be less than the detection limit of $0.1 \mathrm{mg}$ lithium per gram polymer, corresponding to less than 0.006 mol Li per mol monomer.

Preparation of PIL Fibers. To prepare spinning solutions, PIL and PVP were mixed with DMF and $\mathrm{MeOH}$ using a magnetic stirrer heated to $35^{\circ} \mathrm{C}$. Solutions were transferred to a $2 \mathrm{~mL}$ syringe and mounted on the syringe pump in a spinning machine (Professional Electrospinner, Yflow, Spain). A positive high voltage was connected via an alligator clip to a needle with an inner diameter of $0.5 \mathrm{~mm}$ and outer diameter of $1 \mathrm{~mm}$. The collecting plate was covered with an aluminum foil and connected to a ground. Process parameters were fixed at a voltage of $20 \mathrm{kV}$, flow rate of $1.2 \mathrm{~mL} / \mathrm{h}$, and distance from tip to collector of $10 \mathrm{~cm}$. The temperature was $23{ }^{\circ} \mathrm{C}$ and the humidity $40-50 \%$.

Characterization. SEM was performed on a GEMINI LEO 1550 microscope at $3 \mathrm{kV}$; samples were coated with a thin layer of gold and palladium before examination. SAXS was carried using a Nanostar (Bruker AXS, Karlsruhe, Germany) device, equipped with a SIEMENS KFF CU 2K-90 X-ray tube, operating at $40 \mathrm{kV}$ and 100 $\mathrm{mA}$, and generating an X-ray beam with a wavelength of $1.5418 \AA(\mathrm{Cu}$ $\mathrm{K} \alpha$ radiation) and a focal spot size of $550 \mu \mathrm{m}$. A silver behenate standard was used to calculate beam center and the exact sample-todetector distance. 2D scattering plots were corrected for background, transmission, and empty capillary.

Surface tension was measured using the drop profile analysis tensiometer (PAT-1, SINTERFACE Technologies). Conductivity of solutions was measured using an InLab 751-4mm sensor connected to a conductivity meter (Mettler Toledo). The density of the sample at $25{ }^{\circ} \mathrm{C}$ was determined in a density oscillation tube (DMA 5000M, Anton Paar, Graz). The viscosity at $25{ }^{\circ} \mathrm{C}$ was measured and evaluated with an Automated Microviscometer (AMVn, Anton Paar, Graz), a capillary with a diameter of $3 \mathrm{~mm}$ at $70^{\circ}$ angle and $2 \times 6$ repeated measurements.

Molecular weight of the polymers was measured using analytical ultracentrifugation (AUC), performed on an Optima XLI centrifuge (Beckman Coulter, Palo Alto CA) and Rayleigh interference optics at $25{ }^{\circ} \mathrm{C}$ and a speed of $60000 \mathrm{rpm}$. The sedimentation coefficient distributions were evaluated with the least squares $g^{*}(s)$ evaluation implemented in the software SEDFIT (version $15.01 b^{69}$ ). For the equilibrium experiments, concentrations between 1 and $5 \mathrm{mg} / \mathrm{mL}$ were analyzed at different speeds starting from $5000 \mathrm{rpm}$ up to 10000 $\mathrm{rpm}$ for the $110 \mathrm{kDa}$ PIL and at $3500 \mathrm{rpm}$ for the $315 \mathrm{kDa}$ PIL. Data were evaluated with the program MSTAR (Kristian Schilling, Nanolytics, Germany). ${ }^{70}$

\section{ASSOCIATED CONTENT}

\section{S Supporting Information}

The Supporting Information is available free of charge on the ACS Publications website at DOI: 10.1021/acs.macromol.9b00691. 
${ }^{1} \mathrm{H}$ NMR spectrum of the PIL and its structure, pictures of the spun sheet, SAXS of PVP and MIX-25, and TGA of the fibers, PVP and PIL (PDF)

\section{AUTHOR INFORMATION}

\section{Corresponding Author}

*E-mail: Elinor.josef@mpikg.mpg.de.

\section{ORCID}

Elinor Josef: 0000-0002-9744-7399

Ryan Guterman: 0000-0003-3231-6176

\section{Notes}

The authors declare no competing financial interest.

\section{ACKNOWLEDGMENTS}

The authors thank Aliyar Javadi (surface tension), Antje Völkel (AUC and viscosity), and Daniel Werner (SAXS measurements).

\section{REFERENCES}

(1) Chew, S.; Wen, Y.; Dzenis, Y.; Leong, K. The Role of Electrospinning in the Emerging Field of Nanomedicine. Curr. Pharm. Des. 2006, 12, 4751-4770.

(2) Sill, T. J.; von Recum, H. A. Electrospinning: Applications in Drug Delivery and Tissue Engineering. Biomaterials 2008, 29, 19892006

(3) Li, Z.; Wang, C. One Dimensional Nanostructures Electrospinning Technique and Unique Nanofibers; Springer, 2013.

(4) Xue, J.; Xie, J.; Liu, W.; Xia, Y. Electrospun Nanofibers: New Concepts, Materials, and Applications. Acc. Chem. Res. 2017, 50, 1976-1987.

(5) Visser, J.; Melchels, F. P. W.; Jeon, J. E.; Van Bussel, E. M.; Kimpton, L. S.; Byrne, H. M.; Dhert, W. J. A.; Dalton, P. D.; Hutmacher, D. W.; Malda, J. Reinforcement of Hydrogels Using Three-Dimensionally Printed Microfibres. Nat. Commun. 2015, 6, 6933.

(6) Kenry; Lim, C. T. Nanofiber Technology: Current Status and Emerging Developments. Prog. Polym. Sci. 2017, 70, 1-17.

(7) Lu, X.; Wang, C.; Wei, Y. One-Dimensional Composite Nanomaterials: Synthesis by Electrospinning and Their Applications. Small 2009, 5, 2349-2370.

(8) Ngiam, M.; Ramakrishna, S.; Raghunath, M.; Chan, C. K. Nanofiber Patent Landscape. Recent Pat. Nanotechnol. 2007, 1, 137144.

(9) Dzenis, Y. Spinning Continuous Fibers for Nanotechnology. Science 2004, 304, 1917-1919.

(10) Engel, Y.; Schiffman, J. D.; Goddard, J. M.; Rotello, V. M. Nanomanufacturing of Biomaterials. Mater. Today 2012, 15, 478485 .

(11) Wang, H.-G.; Yuan, S.; Ma, D.-L.; Zhang, X.-B.; Yan, J.-M. Electrospun Materials for Lithium and Sodium Rechargeable Batteries: From Structure Evolution to Electrochemical Performance. Energy Environ. Sci. 2015, 8, 1660-1681.

(12) Yu, H.-Y.; Wang, C.; Abdalkarim, S. Y. H. Cellulose Nanocrystals/Polyethylene Glycol as Bifunctional Reinforcing/ Compatibilizing Agents in Poly(Lactic Acid) Nanofibers for Controlling Long-Term in Vitro Drug Release. Cellulose 2017, 24, 4461-4477.

(13) Yu, H.-F.; Kao, S.-Y.; Lu, H.-C.; Lin, Y.-F.; Feng, H.; Pang, H.W.; Vittal, R.; Lin, J.-J.; Ho, K.-C. Electrospun Nanofibers Composed of Poly(Vinylidene Fluoride-Co-Hexafluoropropylene) and Poly(Oxyethylene)-Imide Imidazolium Tetrafluoroborate as Electrolytes for Solid-State Electrochromic Devices. Sol. Energy Mater. Sol. Cells 2018, 177, 32-43.

(14) Li, H.-Y.; Liu, Y.-L. Polyelectrolyte Composite Membranes of Polybenzimidazole and Crosslinked Polybenzimidazole-Polybenzox- azine Electrospun Nanofibers for Proton Exchange Membrane Fuel Cells. J. Mater. Chem. A 2013, 1, 1171-1178.

(15) Theron, S. A.; Zussman, E.; Yarin, A. L. Experimental Investigation of the Governing Parameters in the Electrospinning of Polymer Solutions. Polymer 2004, 45, 2017-2030.

(16) Greiner, A.; Wendorff, J. H. Electrospinning: A Fascinating Method for the Preparation of Ultrathin Fibers. Angew. Chem., Int. Ed. 2007, 46, 5670-5703.

(17) Son, W. K.; Youk, J. H.; Lee, T. S.; Park, W. H. The Effects of Solution Properties and Polyelectrolyte on Electrospinning of Ultrafine Poly(Ethylene Oxide) Fibers. Polymer 2004, 45, 29592966.

(18) Pillay, V.; Dott, C.; Choonara, Y. E.; Tyagi, C.; Tomar, L.; Kumar, P.; du Toit, L. C.; Ndesendo, V. M. K. A Review of the Effect of Processing Variables on the Fabrication of Electrospun Nanofibers for Drug Delivery Applications. J. Nanomater. 2013, 2013, 1-22.

(19) Reneker, D. H.; Yarin, A. L. Electrospinning Jets and Polymer Nanofibers. Polymer 2008, 49, 2387-2425.

(20) Hohman, M. M.; Shin, M.; Rutledge, G.; Brenner, M. P. Electrospinning and Electrically Forced Jets. I. Stability Theory. Phys. Fluids 2001, 13, 2201-2220.

(21) Cadafalch Gazquez, G.; Smulders, V.; Veldhuis, S.; Wieringa, P.; Moroni, L.; Boukamp, B.; Ten Elshof, J. Influence of Solution Properties and Process Parameters on the Formation and Morphology of YSZ and NiO Ceramic Nanofibers by Electrospinning. Nanomaterials 2017, 7, 16 .

(22) Haider, A.; Haider, S.; Kang, I.-K. A Comprehensive Review Summarizing the Effect of Electrospinning Parameters and Potential Applications of Nanofibers in Biomedical and Biotechnology. Arabian J. Chem. 2018, 11, 1165-1188.

(23) Lubasova, D.; Spanek, R. Study of Relationships Between Morphology of Polyvinylbutyral Nanofibers and Solvents Properties Using a Predictive Numerical Model. J. Comput. Theor. Nanosci. 2017, 14, 2802-2811.

(24) McKee, M. G.; Hunley, M. T.; Layman, J. M.; Long, T. E. Solution Rheological Behavior and Electrospinning of Cationic Polyelectrolytes. Macromolecules 2006, 39, 575-583.

(25) Séon-Lutz, M.; Couffin, A.-C.; Vignoud, S.; Schlatter, G.; Hébraud, A. Electrospinning in Water and in Situ Crosslinking of Hyaluronic Acid/Cyclodextrin Nanofibers: Towards Wound Dressing with Controlled Drug Release. Carbohydr. Polym. 2019, 207, 276287.

(26) Hayati, I.; Bailey, A. I.; Tadros, T. F. Investigations into the Mechanisms of Electrohydrodynamic Spraying of Liquids. J. Colloid Interface Sci. 1987, 117, 205-221.

(27) Kim, B.; Park, H.; Lee, S.-H.; Sigmund, W. M. Poly(Acrylic Acid) Nanofibers by Electrospinning. Mater. Lett. 2005, 59, 829-832.

(28) Shenoy, S. L.; Bates, W. D.; Frisch, H. L.; Wnek, G. E. Role of Chain Entanglements on Fiber Formation during Electrospinning of Polymer Solutions: Good Solvent, Non-Specific Polymer-Polymer Interaction Limit. Polymer 2005, 46, 3372-3384.

(29) Klossner, R. R.; Queen, H. A.; Coughlin, A. J.; Krause, W. E. Correlation of Chitosan's Rheological Properties and Its Ability to Electrospin. Biomacromolecules 2008, 9, 2947-2953.

(30) Schreiner, C.; Bridge, A. T.; Hunley, M. T.; Long, T. E.; Green, M. D. Segmented Imidazolium Ionenes: Solution Rheology, Thermomechanical Properties, and Electrospinning. Polymer 2017, 114, 257-265.

(31) Jousset, S.; Bellissent, H.; Galin, J. C. Polyelectrolytes of High Charge Density in Organic Solvents. Synthesis and Viscosimetric Behavior. Macromolecules 1998, 31, 4520-4530.

(32) Ono, T.; Ohta, M.; Sada, K. Ionic Polymers Act as Polyelectrolytes in Nonpolar Media. ACS Macro Lett. 2012, 1 , $1270-1273$

(33) Radeva, T. Physical Chemistry of Polyelectrolytes; Radeva, T., Ed.; CRC Press, 2001; Vol. 99.

(34) Waigh, T. A.; Ober, R.; Williams, C. E.; Galin, J.-C. Semidilute and Concentrated Solutions of a Solvophobic Polyelectrolyte in Nonaqueous Solvents. Macromolecules 2001, 34, 1973-1980. 
(35) Smolyakov, G.; Catala, J.-M.; Kutsevol, N.; Rawiso, M. Influence of the Nature of Counterions and Solvent on the Structure of PSS Solutions. In Modern Problems of Molecular Physics; Bulavin, L. A., Chalyi, A. V., Eds.; Springer International Publishing: Cham, 2018; pp 133-147.

(36) Dou, S.; Colby, R. H. Charge Density Effects in Salt-Free Polyelectrolyte Solution Rheology. J. Polym. Sci., Part B: Polym. Phys. 2006, 44, 2001-2013.

(37) Liu, X.; Hu, O.; Tong, Z. Viscosity Behavior of Polyelectrolyte Copolymers Containing Sulfonate Groups in Mixed Organic Solvents. J. Polym. Sci., Part B: Polym. Phys. 1997, 35, 1433-1438.

(38) Essafi, W.; Spiteri, M.-N.; Williams, C.; Boue, F. Hydrophobic Polyelectrolytes in Better Polar Solvent. Structure and Chain Conformation As Seen by SAXS and SANS. Macromolecules 2009, $42,9568-9580$.

(39) Antonietti, M.; Foerster, S.; Zisenis, M.; Conrad, J. Solution Viscosity of Polyelectrolyte-Surfactant Complexes: Polyelectrolyte Behavior in Nonaqueous Solvents. Macromolecules 1995, 28, 22702275.

(40) Rošic, R.; Pelipenko, J.; Kristl, J.; Kocbek, P.; Bešter-Rogač, M.; Baumgartner, S. Physical Characteristics of Poly (Vinyl Alcohol) Solutions in Relation to Electrospun Nanofiber Formation. Eur. Polym. J. 2013, 49, 290-298.

(41) Combet, J. Polyelectrolytes and Small Angle Scattering. EPJ Web Conf. 2018, 188, 03001.

(42) Pedersen, J. S.; Schurtenberger, P. Scattering Functions of Semidilute Solutions of Polymers in a Good Solvent. J. Polym. Sci. 2004, 42, 3081-3094.

(43) Colby, R. H. Structure and Linear Viscoelasticity of Flexible Polymer Solutions: Comparison of Polyelectrolyte and Neutral Polymer Solutions. Rheol. Acta 2010, 49, 425-442.

(44) Regev, O.; Khalfin, R.; Zussman, E.; Cohen, Y. About the Albumin Structure in Solution and Related Electro-Spinnability Issues. Int. J. Biol. Macromol. 2010, 47, 261-265.

(45) Liu, F.; Nishikawa, T.; Shimizu, W.; Sato, T.; Usami, H.; Amiya, S.; Ni, Q.-Q.; Murakami, Y. Preparation of Fully Hydrolyzed Polyvinyl Alcohol Electrospun Nanofibers with Diameters of Sub-200 Nm by Viscosity Control. Text. Res. J. 2012, 82, 1635-1644.

(46) Yuan, J.; Antonietti, M. Poly(Ionic Liquid)s: Polymers Expanding Classical Property Profiles. Polymer 2011, 52, 1469-1482.

(47) Isik, M.; Porcarelli, L.; Lago, N.; Zhu, H.; Forsyth, M.; Mecerreyes, D. Proton Conducting Membranes Based on Poly(Ionic Liquids) Having Phosphonium Counter-Cations. Macromol. Rapid Commun. 2018, 39, 1700627.

(48) Montolio, S.; Abarca, G.; Porcar, R.; Dupont, J.; Burguete, M. I.; García-Verdugo, E.; Luis, S. V. Hierarchically Structured Polymeric Ionic Liquids and Polyvinylpyrrolidone Mat-Fibers Fabricated by Electrospinning. J. Mater. Chem. A 2017, 5, 9733-9744.

(49) Qian, W.; Texter, J.; Yan, F. Frontiers in Poly(Ionic Liquid)s: Syntheses and Applications. Chem. Soc. Rev. 2017, 46, 1124-1159.

(50) Ajjan, F. N.; Ambrogi, M.; Tiruye, G. A.; Cordella, D.; Fernandes, A. M.; Grygiel, K.; Isik, M.; Patil, N.; Porcarelli, L.; Rocasalbas, G.; et al. Innovative Polyelectrolytes/Poly(Ionic Liquid)s for Energy and the Environment. Polym. Int. 2017, 66, 1119-1128.

(51) Mecerreyes, D. Polymeric Ionic Liquids: Broadening the Properties and Applications of Polyelectrolytes. Prog. Polym. Sci. 2011, 36, 1629-1648.

(52) Yuan, J.; Mecerreyes, D.; Antonietti, M. Poly(Ionic Liquid)s: An Update. Prog. Polym. Sci. 2013, 38, 1009-1036.

(53) Einert, M.; Wessel, C.; Badaczewski, F.; Leichtweiß, T.; Eufinger, C.; Janek, J.; Yuan, J.; Antonietti, M.; Smarsly, B. M.; Eufi, C.; et al. Nitrogen-Doped Carbon Electrodes: Influence of Microstructure and Nitrogen Configuration on the Electrical Conductivity of Carbonized Polyacrylonitrile and Poly(ionic liquid) Blends. Macromol. Chem. Phys. 2015, 216, 1930-1944.

(54) Pang, H.-W.; Yu, H.-F.; Huang, Y.-J.; Li, C.-T.; Ho, K.-C. Electrospun Membranes of Imidazole-Grafted PVDF-HFP Polymeric Ionic Liquids for Highly Efficient Quasi-Solid-State Dye-Sensitized Solar Cells. J. Mater. Chem. A 2018, 6, 14215-14223.
(55) Yuan, J.; Márquez, A. G.; Reinacher, J.; Giordano, C.; Janek, J.; Antonietti, M. Nitrogen-Doped Carbon Fibers and Membranes by Carbonization of Electrospun Poly(Ionic Liquid)S. Polym. Chem. 2011, 2, 1654-1657.

(56) Chen, H.; Elabd, Y. A. Polymerized Ionic Liquids: Solution Properties and Electrospinning. Macromolecules 2009, 42, 3368-3373.

(57) Ignatova, M.; Starbova, K.; Markova, N.; Manolova, N.; Rashkov, I. Electrospun Nano-Fibre Mats with Antibacterial Properties from Quaternised Chitosan and Poly(Vinyl Alcohol). Carbohydr. Res. 2006, 341, 2098-2107.

(58) Meng, X.; Perry, S. L.; Schiffman, J. D. Complex Coacervation: Chemically Stable Fibers Electrospun from Aqueous Polyelectrolyte Solutions. ACS Macro Lett. 2017, 6, 505-511.

(59) Eda, G.; Liu, J.; Shivkumar, S. Flight Path of Electrospun Polystyrene Solutions: Effects of Molecular Weight and Concentration. Mater. Lett. 2007, 61, 1451-1455.

(60) Zhao, Q.; Fellinger, T.-P.; Antonietti, M.; Yuan, J. A Novel Polymeric Precursor for Micro/Mesoporous Nitrogen-Doped Carbons. J. Mater. Chem. A 2013, 1, 5113.

(61) Fong, H.; Chun, I.; Reneker, D. H. Beaded Nanofibers Formed during Electrospinning. Polymer 1999, 40, 4585-4592.

(62) Bhardwaj, N.; Kundu, S. C. Electrospinning: A Fascinating Fiber Fabrication Technique. Biotechnol. Adv. 2010, 28, 325-347.

(63) Shi, X.; Zhou, W.; Ma, D.; Ma, Q.; Bridges, D.; Ma, Y.; Hu, A. Electrospinning of Nanofibers and Their Applications for Energy Devices. J. Nanomater. 2015, 2015, 1-20.

(64) Wendorff, J. H.; Agarwal, S.; Greiner, A. Electrospinning: Materials, Processing, and Applications; WILEY-VCH Verlag, 2012.

(65) Rošic, R.; Pelipenko, J.; Kocbek, P.; Baumgartner, S.; BešterRogač, M.; Kristl, J. The Role of Rheology of Polymer Solutions in Predicting Nanofiber Formation by Electrospinning. Eur. Polym. J. 2012, 48, 1374-1384.

(66) Agrahari, V.; Agrahari, V.; Meng, J.; Mitra, A. K. Electrospun Nanofibers in Drug Delivery: Fabrication, Advances, and Biomedical Applications. In Emerging Nanotechnologies for Diagnostics, Drug Delivery and Medical Devices; Mitra, A. K., Cholkar, K., Mandal, A.; Micro and Nano Technologies, Eds.; Elsevier: Boston, 2017; pp 189215.

(67) Wyatt, N. B.; Liberatore, M. W. Rheology and Viscosity Scaling of the Polyelectrolyte Xanthan Gum. J. Appl. Polym. Sci. 2009, 114, 4076-4084.

(68) Peterlin, A. Small Angle Scattering by a Three Component System. Makromol. Chem. 1965, 87, 152-165.

(69) Schuck, P. Size-Distribution Analysis of Macromolecules by Sedimentation Velocity Ultracentrifugation and Lamm Equation Modeling. Biophys. J. 2000, 78, 1606-1619.

(70) Cölfen, H.; Harding, S. E. MSTARA and MSTARI: Interactive PC Algorithms for Simple, Model Independent Evaluation of Sedimentation Equilibrium Data. Eur. Biophys. J. 1997, 25, 333-346. 